\title{
Predictive regulation of cardiovascular system on emergence of auditory-motor interaction in young infants
}

\author{
Yuta Shinya $^{1 *}$, Kensuke $\mathrm{Oku}^{2}$, Hama Watanabe ${ }^{1}$, Gentaro Taga ${ }^{1}, \&$ Shinya Fujii ${ }^{2}$ \\ ${ }^{1}$ Graduate School of Education, The University of Tokyo, Tokyo, Japan \\ ${ }^{2}$ Faculty of Environment and Information Studies, Keio University, Kanagawa, Japan
}

*Corresponding author: Yuta Shinya, E-mail: shinya@p.u-tokyo.ac.jp

\begin{abstract}
Humans develop auditory-motor interaction to produce a variety of rhythmic sounds using body movements, which are often produced and amplified with tools, such as drumming. The extended production of sounds allows us to express a wide range of emotions, accompanied by physiological changes. According to previous studies, even young infants exhibit movements in response to auditory feedback. However, their exhibition of physiological adaptation on emergence of auditory-motor interaction is unclear. We investigated the heart rate change associated with auditory feedback to spontaneous limb movements in 3-month-old infants. The results showed that, in response to the auditory feedback, infants begin to increase heart rate more selectively immediately before the timing of the feedback. Furthermore, they gradually suppress the peak intensity of the heart rate increase through auditory-motor experience. These findings suggest that emergence of auditory-motor interaction in young infants involves predictive regulation to implicitly maintain homeostasis in the cardiovascular system. The predictive regulation, which is referred to as allostasis, may contribute to the prolonged sound production and provide a developmental basis for more sophisticated goal-directed behavior of producing rhythmic sounds.
\end{abstract}

Keywords: allostasis, auditory-motor interaction, autonomic nervous system, infants, motor development, musicality 


\section{Introduction}

Humans produce a variety of sounds not only by using their vocal organs, but also their body movements, such as through drumming. The beat is often produced and amplified with tools such as musical instruments (Patel 2014); for example, tool-assisted rhythmic drumming is assumed to be unique to humans, except for only a few species of animals (e.g. palm cockatoo) (Heinsohn et al. 2017). The extended production of sounds allows us to express various rhythms and melodies to induce a wide range of emotions and feelings, accompanied by physiological as well as behavioral changes (Blood and Zatorre 2001; Habibi and Damasio 2014; Scherer and Zentner 2001). Furthermore, in the long term, these changes associated with music activities are assumed to play a crucial role in subjective well-being and health through homeostatic processes, such as stress reduction (Bainbridge et al. 2020; Chanda and Levitin 2013; Cirelli and Trehub 2020; de Witte et al. 2020; Tramo et al. 2011).

Recent cognitive neuroscientific research of music shows that both perception and production of music require the interaction between auditory and motor systems (Zatorre et al. 2007). Especially, the integration of spatial, auditory, and motor information has been assumed to be essential in producing rhythmic sounds by controlling movements, such as drumming. Humans acquire universal musicality, including the ability to produce rhythmic sounds, which is often amplified using musical instruments (Kotz et al. 2018; Savage et al. 2015). However, the emergence of these auditory-motor interactions in the acquisition of sound production in early development remains unclear.

As opposed to sound perception, which indicates the early ability to process musical sounds (Hannon and Johnson 2005; Perani et al. 2010; Phillips-Silver and Trainor, 2005; Winkler et al. 2009), the development of sound production in infancy is directly revealed by only a few studies. Infants typically produce rhythmic vocalization (e.g. babbling) and movement (e.g. banging, shaking) in the first few years of life (Dolata et al. 2008; Ejiri 1998; Thelen 1979; Cirelli and Trehub 2019; Zentner and Eerola 2010; Fujii et al. 2014). For example, Zentner and Eerola (2010) reported that 5- to 24-months old infants engaged in rhythmic limb movements when listening to music. Fujii et al. (2014) reported that three- to four-months-old infants are already primed to interact with music via limb movements and vocalizations. Therefore, it is possible that even young infants develop the precursors of auditory-motor interactions, allowing for more sophisticated music production such as dancing or drumming in later developmental stages.

Previous studies investigating the development of memory or sense of self-agency have shown that young infants would enhance movements in response to contingent changes in the 
environment including auditory sounds (Bahrick and Watson 1985; DeCasper and Fifer 1980; Rochat and Striano 1999; Rovee-Collier 1999; Watanabe et al. 2011). For example, in a situation where sucking induced presentation of mother's speech or face, new-borns increased their oral movements to induce the presentation (DeCasper and Fifer 1980). Similarly, in the sucking task, 2-month-old infants showed modulation of their oral movements in conditions where the pitch of the contingent auditory feedback varied depending on the pressure of their sucking (Rochat and Striano 1999). Furthermore, in the mobile task, supine young infants with a limb connected to an overhead mobile, selectively increased the limb's movements to induce audio-visual stimulation from the mobile (Rovee-Collier 1999; Watanabe et al. 2011).

These findings suggest that young infants are motivated to increase their body movements to produce sounds in situations where their body movements could induce or be converted to auditory information. Noteworthily, in this period, it would be challenging for infants to inhibit and regulate spontaneous movements automatically generated by subcortical systems (i.e. central pattern generator in brainstem and spinal cord) (Watanabe et al. 2011; Prechtl 1997). Thus, the findings are assumed to be evidence for the precursor of goal-directed behavior due to cortical development, providing a base for the acquisition of music production.

As opposed to behavioral adaptation (i.e. enhancement of motor activities), few studies investigated the physiological activities through the auditory-motor interaction in early infancy. Reportedly, foetus (Lecanuet et al. 1992) and young infants (Morrongiello and Clifton 1984; Porges et al. 1973) display heart rate deceleration when exposed to auditory stimuli, which may be a function of orienting response (Graham and Clifton 1966) and sustained attention (Richards and Casey 1991). Also, such cardiovascular response may depend on predictability of the timing of auditory stimuli (Hajack et al. 2003; 2004). Given that the auditory-motor interaction allows prediction of auditory feedback in response to movement (Zatorre et al. 2007), it is possible that the dynamic change in cardiovascular system may occur in the early process of auditory-motor interaction in young infants.

Moreover, as a notable aspect of music, its production and perception elicit feelings and emotions which involve arousal, stress regulation, and behavioral and attentional changes (Habibi and Damasio 2014; Scherer and Zentner 2001). Especially, auditory-motor synchronization has been reported to decrease the physiological load in cardiovascular system, even in the absence of musical component (Bood et al. 2013; Chaisurin et al. 2020; Trappe 2010). This predictive mode to maintain homeostasis regulation via behavioral and physiological changes is considered as 'allostasis' (Sterling 2012). Therefore, we speculate that the auditory-motor interaction in young infants would accompany not only just behavioral 
adaptation, but also allostatic regulation in cardiovascular system.

To the best of our knowledge, no study has revealed the dynamic change in cardiovascular activity in response to auditory feedback associated with movement in infants. A few studies investigated more global changes of parasympathetic activities, indexed by respiratory sinus arrhythmia (RSA) (Task Force 1996; Denver et al. 2007), in the mobile paradigm using audio-visual feedback (Haley et al. 2008; Sullivan 2016). Haley et al. (2008), reported that, 3-month-old infant learners showed greater suppression of RSA than the non-learners. Alternatively, Sullivan (2016) reported that, in 5-month-old infants, RSA did not vary significantly over sessions, and was not associated with learning status. Nevertheless, more localized, and dynamic cardiovascular changes associated with auditory-motor experience in young infants remains unclear.

By focusing on the physiological aspects, the current study expands the previous observation on emergence of auditory-motor interaction in early infancy (Bahrick and Watson 1985; DeCasper and Fifer 1980; Rochat and Striano 1999; Rovee-Collier 1999; Watanabe et al. 2011). To reveal the physiological adaptation in this context, we investigated heart rate as well as behavioral change through auditory feedback to limb movements. Considering the role of sound production and perception in achieving homeostasis via arousal and stress regulation (Bainbridge et al. 2020; Chanda and Levitin 2013; Cirelli and Trehub, 2020; de Witte et al. 2020; Tramo et al. 2011; Sterling 2012), we hypothesized that the heart rate change related to limb movements inducing auditory feedback may be gradually regulated by changes in limb movements during auditory-motor integration progress. 


\section{Methods}

\section{Participants}

Thirty 3-month-old term infants (10 females) participated in this study. The sample excluded preterm/low-birth weight infants or infants with medical problems. The mean age of the infants was 99.77 days (range: $90-110$ days, $\mathrm{SD}=5.54$ days). Twenty-seven other infants participated but did not complete the experiment due to excessive crying $(n=25)$ and drowsiness $(n=2)$. Additional 10 infants completed the experiment, but were excluded because their excessive fussiness was soothed by the experimenter occasionally. The study was approved by the ethical committee of Life Science Research Ethics and Safety, The University of Tokyo, and was conducted in accordance with standards specified in the 1964 Declaration of Helsinki. Written informed consent was obtained from the participants' parents.

\section{Apparatus and stimuli}

Infants were studied in the laboratory at the Department of Physical and Health Education at the Graduate School of Education, the University of Tokyo. During the experiment each infant was positioned in a supine position on a baby mattress $(120 \mathrm{~cm} \times 70 \mathrm{~cm})$.

Four virtual drum-kit sensors with accelerometer and gyroscope (Freedrum, Sweden; Figure $1 a$ ) were put on each limb of the participants to record the timing of the velocity and acceleration of movements exceeding a threshold. When the threshold was exceeded during Test phase, auditory stimuli was immediately fed to an infant from two speakers (Bose) about $40 \mathrm{~cm}$ near their ears. The auditory stimuli produced by each limb's sensor corresponded to drum sounds with different heights (i.e., high Tom, mid Tom, low Tom, floor Tom; Figure 1b).

(a)

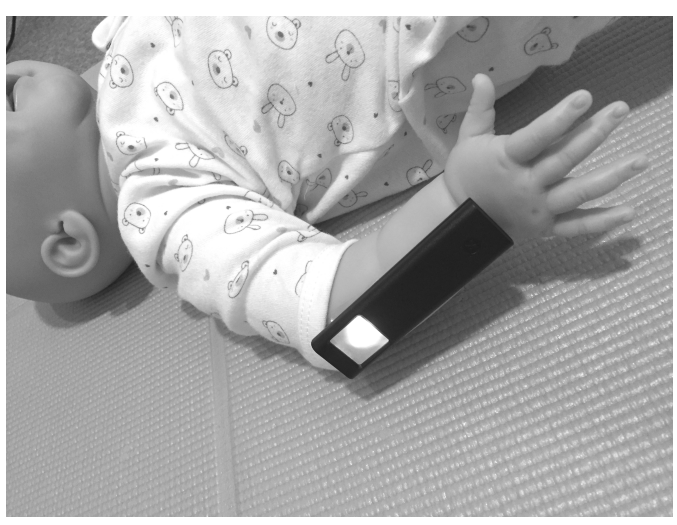

(b)

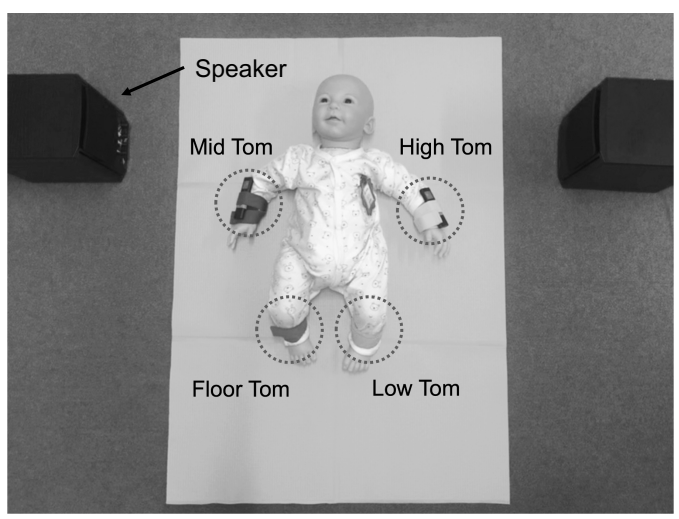

Figure 1. Experimental setup: (a) an infant wearing a virtual drum kit sensor (Freedrum, Sweden) on the right arm; (b) the sounds produced by each limb's sensor correspond to drum sounds of different heights. 
For heart rate recording, we used a wireless multi-telemeter (WEB-1000, Nihon Koden) to obtain the Electrocardiograph (ECG) data. The ECG data were measured from three disposable paediatric ECG electrodes (NC Vitrode, Nihon Koden) using lead II (right collarbone and lower left rib), grounded at the left collarbone. The sampling rate was $1000 \mathrm{~Hz}$.

Additionally, a single video camera (Handycam HDR-CX675, SONY) was used to record the movements, faces, voices, and status of the sensors to assess infants' behavioral status and maintain records.

\section{Experimental procedure}

Infants were positioned on their backs on the baby mattress before commencing the experiment. Four virtual drum-kit sensors were attached to the forearms and the lower legs of each infant.

The experimental session consisted of three periods lasting for a total of 12-min: Pre phase (2-min), Test phase (8-min), and Post phase (2-min). During Pre and Post phases, the speakers' volume was muted to not feed the auditory stimulus contingent to the spontaneous limb movements to the infants. During Test phase, infants were able to listen to and induce the auditory feedback through limb movements. For statistical analysis, Test phase was further divided into four two-minute sub-phases (T1, T2, T3, T4).

During this procedure, the caregiver stayed away from the infant's view and the experimenter did not interact with the infant socially (e.g. eye-contact, speech) to avoid distracting the infant. However, when the infants became excessively fussy or upset during Post phase, the experimenter attempted to soothe the infant, in line with previous studies (e.g., Haley et al. 2008).

\section{Data analysis}

\section{Movement measures}

We obtained the onset time of limb movements which exceeded the threshold to induce auditory feedback $(n=7343)$. If the time difference from the previous data within the same limb was less than $100 \mathrm{msec}$, that data was excluded as a double count $(n=543)$. We also detected the period when the sensors had come off from an infant's limb using video data. The timing data related to these were excluded $(n=121)$, and a total of 6679 timing data of the movement were used for analysis. Based on the time series of the timing data, we calculated the frequency of the limb movements and the standard deviation of the time intervals between the limb movements for each 2-min of each infant to assess the changes in the limb movements. 


\section{$\underline{\text { Heart rate measures }}$}

The recorded ECG data were converted into R-wave intervals after manual artifact correction with Heart Rate Variability Analysis Software (Mindware Technologies LTD, US). The corrected R-wave intervals were converted into time series of heart rate.

Power spectral analysis of heart rate variability (HRV) was performed using the Kubios HRV Analysis Software 2.0 (The Biomedical Signal and Medical Imaging Analysis Group, Department of Applied Physics, University of Kuopio, Finland) (Tarvainen et al. 2014). We determined two main oscillations (Task Force 1996; Denver et al. 2007): a low-frequency component (LF, $0.04-0.24 \mathrm{~Hz}$ ), representing both sympathetic and parasympathetic activity related to the baroreflex system; and a high-frequency component ( $\mathrm{HF}, 0.25-1.50 \mathrm{~Hz})$ representing vagal (parasympathetic) activity modulated by respiratory cycles (i.e., RSA). For the high-frequency component, the bandwidth was extended to $0.25-1.50 \mathrm{~Hz}$ from the adult standard of $0.15-0.40 \mathrm{~Hz}$, due to the higher speed of respiration in young infants (Shinya et al. 2016). Both HRV components were calculated by summing power spectral density values over the bandwidth. The ratio of LF to HF was also calculated as an indicator of sympathovagal balance.

In addition to directly revealing the immediate heart rate change due to the contingent auditory feedback of limb movements, we determined the event-related heart rate change (ERHR) by extracting heart rate change for $10 \mathrm{sec}$ before and after each timing for each limbs' acceleration exceeding the threshold (i.e. the onset of auditory feedback during Test phase). The ERHR baseline was adjusted by subtracting the cubic trend of the time series of heart rate for each phase and each participant.

\section{Statistical analysis}

To assess the movement and cardiac measures except for ERHR and the global changes across phases, we conducted a within subjects one-way analysis of variance (ANOVA, phase: Pre, T1, T2, T3, T4, Post).

To assess the statistically significant occurrence of ERHR for each phase, we created surrogate data by randomly extracting data 200 times from the timeseries of each infant's heart rate. For all continuous ERHR data supplied by all participants, we performed linear mixed-effects models (LMM) including the fixed effect of event (i.e., original vs surrogate) and the random effect of participant with intercept to test whether significant difference occurred at each $0.1 \mathrm{sec}$ time point between the original and surrogate ERHRs.

Additionally, to investigate how the amplitude of ERHRs changes across phases, we 
conducted the LMM to predict the mean ERHR for the period 0-2 sec after each timing for each limbs' acceleration exceeding the threshold $(n=6679)$ by phase (Pre, T1, T2, T3, T4, post) as a fixed effect and participant with intercept $(n=30)$ as a random effect.

The LMMs allowed us to assess the occurrence and change of ERHR across phases without assuming that the observations for successive events were independent as a general linear model (e.g., ANOVA) would require (Quené and van den Bergh 2004). Also, the models allowed for significance tests with high statistical power, as the event-related response data were not averaged over phases per participant.

All statistical analyses were performed using R version 4.0.1 (R Development Core Team 2020). The LMMs were developed using the 'Ime4' package (Bates et al. 2015). In repeated-measures ANOVAs and the LMMs, post hoc multiple comparisons were conducted using sequentially rejective Bonferroni procedure. 


\section{Results}

\section{Change of movement and cardiac measures across phases}

Figure 2 shows the changes of the averaged movement (Figure $2 a$ ) and cardiac measures (Figure 2b, $c, d$ ) across 6 phases (i.e., Pre, T1, T2, T3, T4, Post phase).

For the movement frequency related to auditory feedback, we found a significant main effect of phase $\left(F_{5,145}=18.73, p<.001\right)$. Multiple comparisons of phases revealed that the frequency of the movements during Post phase was greater than those during Pre $(p<.001)$ and T1-T4 phase $(p<.001 ; p<.001 ; p=.008 ; p=.036)$. Also, the frequency during T4 was greater than Pre $(p=.003)$ and T1-T3 phase $(p<.001 ; p<.001 ; p=.034)$, indicating that infants increased frequency of their spontaneous limb movements during the whole experiment (Figure $2 a$ ).

(a)

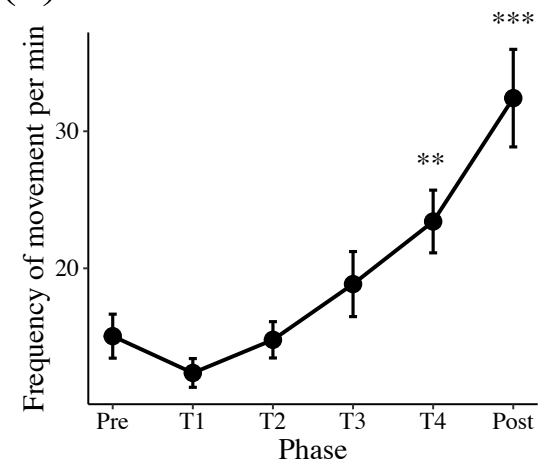

(c)

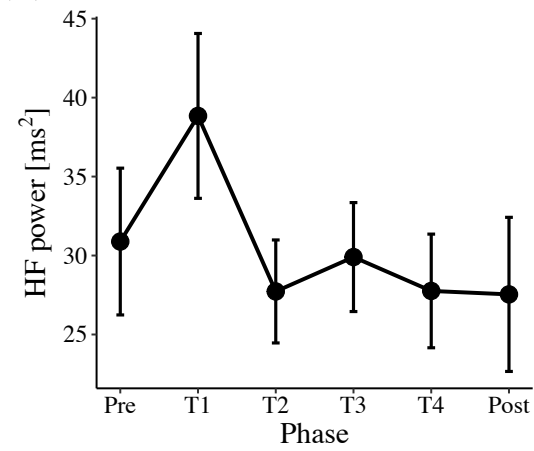

(b)

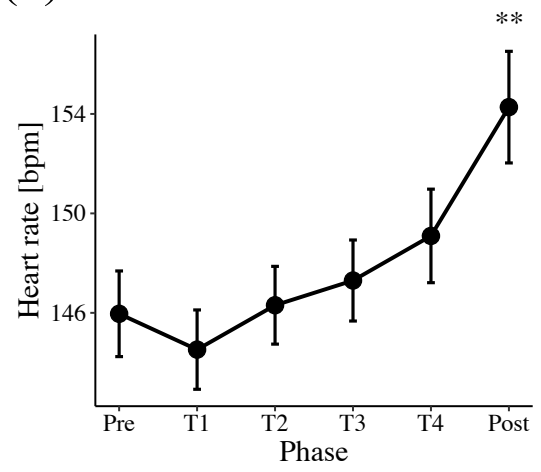

(d)

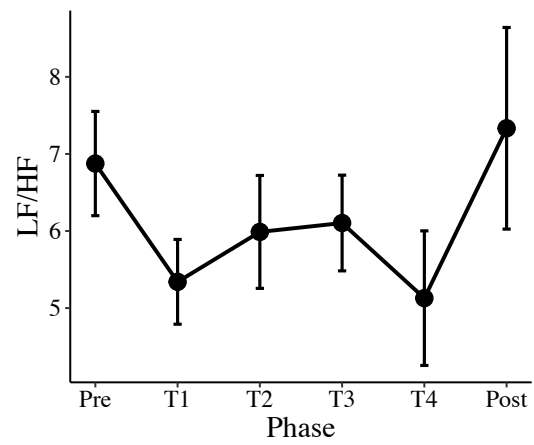

Figure 2. Line plots for changes of movement and cardiac measures across phases: (a) frequency of movement, (b) heart rate, (c) $H F$, and (d) $L F / H F$. Bars represent standard errors. ${ }^{* * *} p<.001,{ }^{* *} p<.01, \stackrel{*}{p}<.05$ (compared to Pre phase).

We observed a significant main effect of phase for heart rate $(\mathrm{HR})\left(F_{5,145}=13.24, p\right.$ $<.001$ ) (Figure 2b). In multiple comparisons, the HR during Post phase was greater than those 
during Pre $(p=.003)$, T1-T4 phase $(p<.001 ; p=.004 ; p=.007 ; p=.020)$. Moreover, the HR during T4 phase was greater than that during T1 phase $(p=.012)$. For HRV measures, only a significant main effect of phase on HF was observed $\left(F_{5,145}=2.48, p=.034\right)$, but there was no significant difference in multiple comparisons (Figure $2 c$ ). These results indicate that, although infants showed increased HR during Post phase, the relatively stable heart rate's and sympathetic/parasympathetic activity's changes were observed from Pre to Test phase.

\section{Comparisons of ERHR between original vs surrogate data}

Figure 3 shows the averaged original and surrogate waveforms of ERHR on Pre, T1-T4, and Post phases for all participants. To determine time point of significant ERHR occurrence, we performed the LMMs including the fixed effect of event (i.e. original vs surrogate) and the random effect of participant with intercept for each 0.1 second of ERHR.
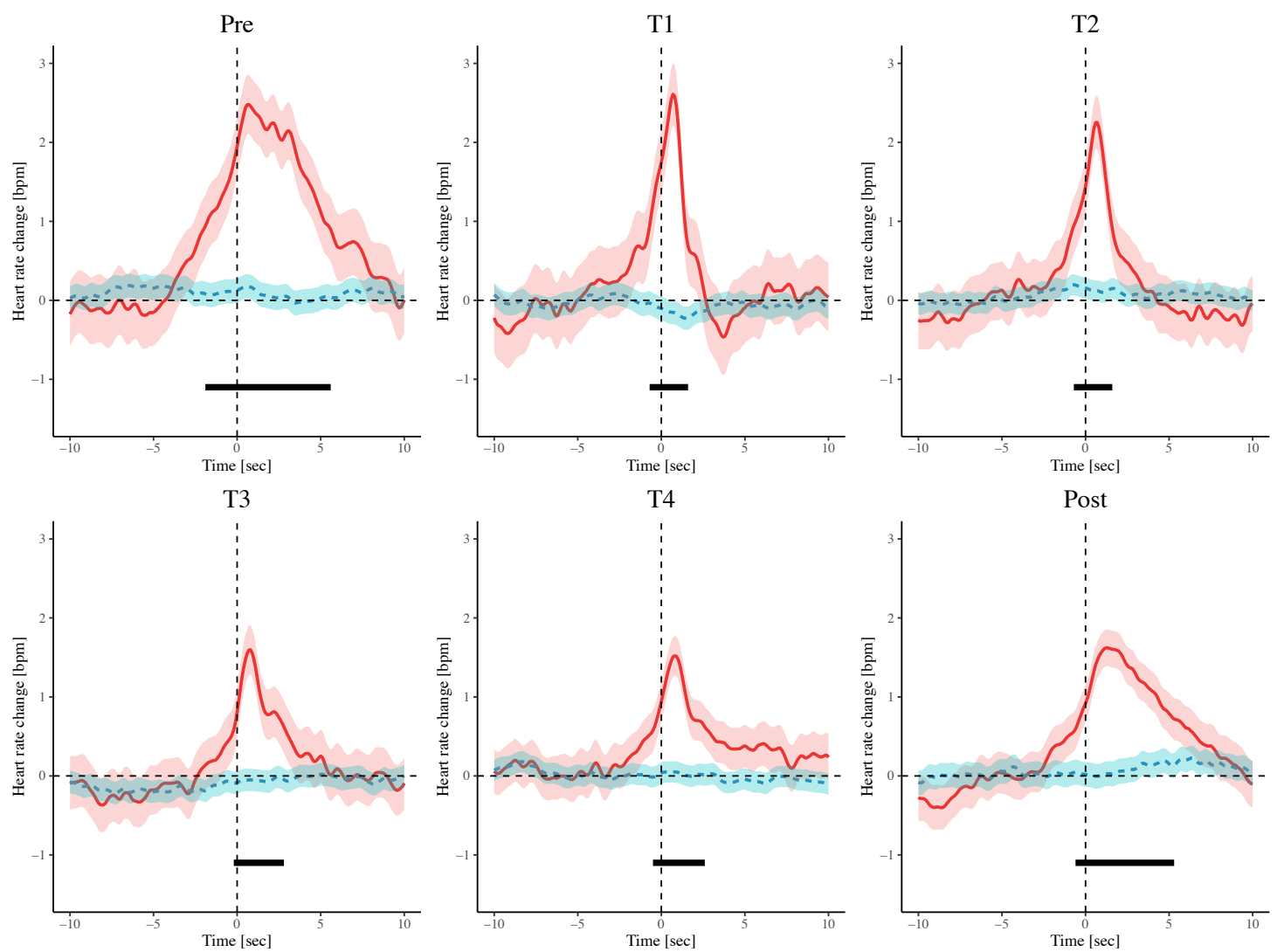

Figure 3. Averaged original (red line) and surrogate (blue line) waveforms of event-related heart rate change for $10 \mathrm{sec}$ before and after timing for each limbs' acceleration exceeding the threshold (i.e. onset of auditory feedback to the limb movement during Test phase) for Pre, T1, T2, T3, T4, and Post phase ( $n=814,716,867,1080,1334,1868$, respectively). Shadows represent 95\% confidence intervals, and black bars represent significant differences between original and surrogate data (adjusted $p<.05$ ). 
The models revealed that the original ERHR was significantly larger than surrogate one during - $1.7 \sim 5.1 \mathrm{sec}$ on Pre phase. Alternatively, on Test phase, the periods of significant difference were relatively shorter compared to Pre phase (T1: $-0.7 \sim 1.6 \mathrm{sec}$; T2: $-0.7 \sim 1.6 \mathrm{sec}$; T3: $-0.2 \sim 2.8 \mathrm{sec} ; \mathrm{T} 4:-0.5 \sim 2.6 \mathrm{sec}$ ). The start point of the significant increase in heart rate on Post phase was closer to the onset, although the end point was similar than that on Pre phase $(-0.6 \sim 5.3 \mathrm{sec})$. These results indicate that experiencing auditory feedback on limb movements led to increase and decrease in heart rate closer to the onset.

\section{Change in ERHR's peak intensity}

Figure 4 shows the change in ERHR's peak intensity (0-2 sec period) across phases. The LMM revealed a significant phase effect $\left(F_{5,6671.8}=6.14, p<.001\right)$. Multiple comparisons indicated that ERHR's peak intensity during Test phase was significantly lower than that during Pre phase (T1: $t=2.88, p=.044$; T2: $t=3.40, p=.008$; T3: $t=4.78, p<.001$; T4: $t=4.95, p$ $<.001)$. Moreover, the peak intensity during Post phase was significantly lower than Pre phase $(t=4.45, p<.001)$.

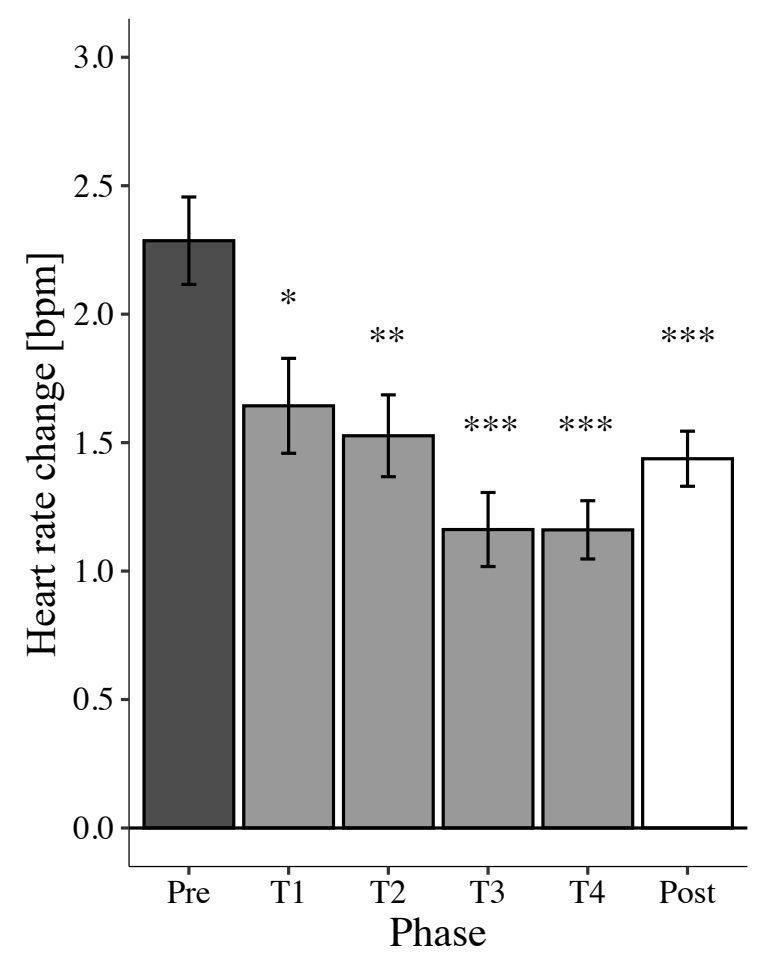

Figure 4. Bar plots of event-related heart rate change's peak intensity (0-2 sec period) across Pre (black), Test (T1-T4; grey), and Post phase (white). Bars represent standard errors. ${ }^{* * *} p$ $<.001,{ }^{* *} p<.01,{ }^{*} p<.05$ (compared to Pre phase). 


\section{Discussion}

The current study is the first to reveal how cardiovascular system would be dynamically regulated on emergence of auditory-motor interaction in early infancy, expanding previous observations regarding behavioral adaptation (Bahrick and Watson 1985; DeCasper and Fifer 1980; Rochat and Striano 1999; Rovee-Collier 1999; Watanabe et al. 2011). We found that, in 3-month-old infants experiencing auditory feedback to spontaneous limb movements, ERHR occurred more selectively at the timing of the auditory feedback. Furthermore, ERHR's peak intensity was gradually inhibited through the experience of auditory feedback. These new findings on physiological adaptation suggest that even young infants regulate cardiovascular system predictively as well as behavioral and attentional changes during the early process of auditory-motor learning.

Several studies investigating memory or sense of self-agency in young infants have reported behavioral adaptation to environmental changes including auditory feedback (i.e. enhancement of motor activities) (Bahrick and Watson 1985; DeCasper and Fifer 1980; Rochat and Striano 1999; Rovee-Collier 1999; Watanabe et al. 2011). Consistent with these findings, we confirmed that 3-month-old infants significantly increased frequency of limb movements inducing auditory feedback from Pre phase to Test and Post phase. This behavioral change has been assumed to reflect increased goal-directed behavior based on predicting visual/auditory feedback (Rochat and Striano 1999; Watanabe et al. 2011; von Hofsten 2004). This interpretation is supported by our findings of the physiological measures.

For global changes of cardiac measures, the infants did not show any significant changes in heart rate and parasympathetic/sympathetic activity (i.e. HF, LF/HF) from Pre phase to Test phase. To reveal a more detailed dynamical change in heart rate, we further investigated ERHR change by averaging time-series of heart rate for $10 \mathrm{sec}$ before and after the onset of auditory feedback to limb movements. As a result, ERHR occurred selectively just before the auditory feedback (Figure 3), and its peak intensity was inhibited gradually through the auditory feedback experience (Figure 4).

Especially, the timing of ERHR occurrence was gradually closer to just before each limbs' acceleration exceeding the threshold during Test phase, with a difference of more than one second between the Pre $(-1.7 \mathrm{sec})$ and T4 $(-0.5 \mathrm{sec})$ phases, suggesting that cardiovascular systems of infants experiencing auditory-motor interaction began to regulate the timing of the heart rate acceleration in anticipation of the auditory feedback to their limb movements. This predictive change in the cardiovascular system may be accompanied by behavioral changes from exploratory movements to goal-directed behavior due to the increased prediction of 
auditory feedback. As a result, decreased activity due to efficient goal-directed behavior may contribute to inhibiting the ERHR's peak intensity. Notably, these changes in ERHR persisted beyond Test phase into Post phase (i.e. the period without auditory feedback), and this persistent effect can be regarded as physiological adaptation to auditory feedback.

We also found that, for the first 2 minutes of the Test phase, infants showed the rapid decrease in ERHR immediately after the auditory feedback. The heart rate deceleration may reflect an orienting response and sustained attention to the auditory stimuli (Lecanuet et al. 1992; Morrongiello and Clifton, 1984; Porges et al. 1973; Graham and Clifton 1966; Richards and Casey 1991). Additionally, the timing of the HR deceleration in ERHR was slower gradually throughout Test phase (T1: $1.6 \mathrm{sec} \sim \mathrm{T} 4: 2.6 \mathrm{sec}$ ). These dynamic changes in heart rate response suggest that infants first pay attention to the auditory feedback of their limb movements and then habituate to the feedback based on their predictions through auditory-motor learning. Nevertheless, rapid heart rate deceleration was still prolonged until the end of Test phase $(2.6 \mathrm{sec})$, compared to Pre phase $(5.1 \mathrm{sec})$. This prolonged regulation of cardiovascular system may reflect a 'moderate' attention to the auditory feedback based on the prediction.

From a physiological viewpoint, the predictive regulation of cardiovascular system observed in this study can be regarded as 'allostasis'. Allostasis, an extended concept of homeostasis meaning 'stability through change', is a dynamic and predictive regulation of internal stability through physiological and behavioral change (Sterling 2012). It may be difficult for young infants to predict auditory feedback associated with movements in a short period, since their ability to coordinate auditory and motor system is still developing. Therefore, relatively large prediction errors could have occurred in our sample, especially on the first 2-min of Test phase. Considering that larger predictive error could induce rapid heart rate deceleration (Hajcak et al. 2003), inhibition of the cardiac response to achieve homeostasis may enhance auditory-motor learning and goal-directed behavior in young infants (Ecker and Gilead 2018; Nagai 2019; Verschure et al. 2014). Indeed, infants showed relatively stable heart rate and parasympathetic/sympathetic activity during Test phase, despite the increased frequency of the limb movement related to the auditory feedback. Therefore, efficient behaviors that place less stress on the cardiovascular system may contribute to prolonged sound production and, in the long term, to acquiring more sophisticated musical behaviors.

Furthermore, our findings are also consistent with several pieces of evidence suggesting that music production and perception may involve arousal and stress regulation accompanied by changes in emotions and feelings (Bainbridge et al. 2020; Blood and Zatorre 2001; Habibi 
and Damasio 2014; Scherer and Zentner 2001; Chanda and Levitin 2013; Cirelli and Trehub 2020; de Witte et al. 2020; Tramo et al. 2011). Especially, it has been emphasized that auditory-motor synchronization plays a role in improving both the physiological load in cardiovascular system and exercise performance (Bood et al. 2013; Chaisurin et al. 2020; Trappe 2010). Reportedly, auditory-motor synchronization is an attention demanding process (Peper et al. 2012; Repp 2005). Thus, Bood et al. (2013) assumed that elevated attention on auditory-motor synchronization may contribute to distraction from fatigue and discomfort. This assumption is compatible with our interpretation of predictive regulation of cardiovascular system in young infants.

The current study had several limitations. First, considering that the average intervals of the limb movements were about $2 \mathrm{sec}$, the overlap of pre- and post-events may have affected ERHR. To control the effects of the frequency on ERHR, we investigated statistically significant occurrence of ERHR by comparing original and surrogate data from the timeseries of each infant's heart rate during the same phase. Nevertheless, it should be noted that the inhibition of ERHR's peak intensity may have been partly associated with the overlapping heart rate responses. Second, the current study design did not include a control condition, although it is a pre-post design. Therefore, the possibility that changes in ERHR may have been caused by increased arousal and fatigue over time should be considered in future studies. Also, although the results of the significant increase in the frequency of limb movements compared with baseline suggest that most of our subjects learned the relationship to auditory feedback, the possibility that some did not learn it well enough should also be considered. Third, another limitation of the study is that we evaluated only the timing for any limb's acceleration exceeding the threshold regarding movement measures. We showed the changes in limb movement related to auditory feedback but could not fully demonstrate the possibility that reduced activity amount due to behavioral efficiency may influence ERHR. Thus, future research should investigate the effects of more detailed movement measures on ERHR by using an accelerometer or a 3D motion-capture system.

In conclusion, we revealed that 3-month-old infants regulate cardiovascular system predictively in the early process of auditory-motor interaction. By experiencing auditory feedback to spontaneous limb movements, they began to increase heart rate more selectively just before the timing of the auditory feedback and to gradually suppress the peak intensity of the heart rate increase. The predictive regulation to implicitly maintain homeostasis in the cardiovascular system may contribute to prolonged sound production, providing a developmental basis of acquisition for more sophisticated goal-directed and musical behavior. 


\section{Acknowledgements}

We appreciate all the families for their participation in study. We also thank Kayo Sato, Yoshiko Koda, and Tomoko Yoneyama for their help with running the experiments. This work was supported by the the Center for Early Childhood Development, Education, and Policy Research (Cedep), Graduate School of Education, the University of Tokyo; and the Grants-in-Aid for Scientific Research from the Japan Society for the Promotion of Science and the Ministry of Education Culture, Sports, Science and Technology (16H06525 to GT; 17KT0135 to HW; $20 \mathrm{H} 04092$ to SF; 20K14253 to YS)

\section{Authors contributions}

YS conceived of the study, designed the study, carried out the experiments, analysed the data, performed the statistical analyses, and drafted the manuscript; KO designed the study, carried out the experiments, and participated in the preliminary analysis; HW conceived of the study, designed the study, carried out the experiments, coordinated the study, and critically revised the manuscript; GT conceived of the study, designed the study, coordinated the study, and critically revised the manuscript; SF conceived of the study, designed the study, coordinated the study, and helped draft the manuscript. All authors gave final approval for publication and agree to be held accountable for the work performed therein.

\section{Statements and declarations}

\section{Conflicts of interest}

The authors declare that there are no conflicts of interest, financial or otherwise.

\section{Ethics approval}

The study was approved by the ethical committee of Life Science Research Ethics and Safety, The University of Tokyo, and was conducted in accordance with standards specified in the 1964 Declaration of Helsinki.

\section{Availability of data and material}

The data that support the findings of this study are openly available in Open Science Framework at https://osf.io/bc438/ 


\section{References}

Bahrick LE, Watson JS (1985) Detection of intermodal proprioceptive-visual contingency as a potential basis of self-perception in infancy. Dev Psychol 21:963-973. https://doi.org/10.1037/0012-1649.21.6.963

Bainbridge CM, Bertolo M, Youngers J, et al (2021) Infants relax in response to unfamiliar foreign lullabies. Nat Hum 5:256-264. https://doi.org/10.1038/s41562-020-00963-z

Bates D, Mächler M, Bolker B, Walker S (2015) Fitting Linear Mixed-Effects Models Using lme4. Journal of Statistical Software, Articles 67:1-48. https://doi.org/10.18637/jss.v067.i01

Blood AJ, Zatorre RJ (2001) Intensely pleasurable responses to music correlate with activity in brain regions implicated in reward and emotion. Proc Natl Acad Sci U S A 98:11818-11823. https://doi.org/10.1073/pnas.191355898

Bood RJ, Nijssen M, van der Kamp J, Roerdink M (2013) The power of auditory-motor synchronization in sports: enhancing running performance by coupling cadence with the right beats. PLoS One 8:e70758. https://doi.org/10.1371/journal.pone.0070758

Chaisurin P, Klapajone J, Yaicharoen P (2020) Effects of Synchronous and Asynchronous Music on Heart rate and Perceived Exertion during Aerobic Exercise: Pilot study. Music Med 12:92-99

Chanda ML, Levitin DJ (2013) The neurochemistry of music. Trends Cogn Sci 17:179-193. https://doi.org/10.1016/j.tics.2013.02.007

Cirelli LK, Trehub SE (2019) Dancing to Metallica and Dora: Case Study of a 19-Month-Old. Front Psychol 10:1073. https://doi.org/10.3389/fpsyg.2019.01073

Cirelli LK, Trehub SE (2020) Familiar songs reduce infant distress. Dev Psychol 56:861-868. https://doi.org/10.1037/dev0000917

DeCasper AJ, Fifer WP (1980) Of human bonding: newborns prefer their mothers' voices. Science 208:1174-1176. https://doi.org/10.1126/science.7375928

Denver JW, Reed SF, Porges SW (2007) Methodological issues in the quantification of $\begin{array}{lllll}\text { respiratory } & \text { sinus } & \text { arrhythmia. } & \text { Biol 286-294. }\end{array}$ https://doi.org/10.1016/j.biopsycho.2005.09.005

de Witte M, Spruit A, van Hooren S, et al (2020) Effects of music interventions on stress-related outcomes: a systematic review and two meta-analyses. Health Psychol Rev 14:294-324. https://doi.org/10.1080/17437199.2019.1627897

Dolata JK, Davis BL, Macneilage PF (2008) Characteristics of the rhythmic organization of 
vocal babbling: implications for an amodal linguistic rhythm. Infant Behav Dev 31:422-431. https://doi.org/10.1016/j.infbeh.2007.12.014

Ecker Y, Gilead M (2018) Goal-Directed Allostasis: The Unique Challenge of Keeping Things as They Are and Strategies to Overcome It. Perspect Psychol Sci 13:618-633. https://doi.org/10.1177/1745691618769847

Ejiri K (1998) Relationship between rhythmic behavior and canonical babbling in infant vocal development. Phonetica 55:226-237. https://doi.org/10.1159/000028434

Fujii S, Watanabe H, Oohashi H, et al (2014) Precursors of dancing and singing to music in three- to four-months-old infants. PLoS One 9:e97680. https://doi.org/10.1371/journal.pone.0097680

Graham FK, Clifton RK (1966) Heart-rate change as a component of the orienting response. Psychol Bull 65:305-320. https://doi.org/10.1037/h0023258

Habibi A, Damasio A (2014) Music, feelings, and the human brain. Psychomusicology: Music, Mind, and Brain 24:92-102. https://doi.org/10.1037/pmu0000033

Hajcak G, McDonald N, Simons RF (2003) To err is autonomic: error-related brain potentials, ANS activity, and post-error compensatory behavior. Psychophysiology 40:895-903. https://doi.org/10.1111/1469-8986.00107

Hajcak G, McDonald N, Simons RF (2004) Error-related psychophysiology and negative affect. Brain Cogn 56:189-197. https://doi.org/10.1016/j.bandc.2003.11.001

Haley DW, Grunau RE, Oberlander TF, Weinberg J (2008) Contingency Learning and Reactivity in Preterm and Full-Term Infants at 3 Months. Infancy 13:570-595. https://doi.org/10.1080/15250000802458682

Hannon EE, Johnson SP (2005) Infants use meter to categorize rhythms and melodies: implications for musical structure learning. Cogn Psychol 50:354-377. https://doi.org/10.1016/j.cogpsych.2004.09.003

Heinsohn R, Zdenek CN, Cunningham RB, et al (2017) Tool-assisted rhythmic drumming in palm cockatoos shares key elements of human instrumental music. Sci Adv 3:e1602399. https://doi.org/10.1126/sciadv.1602399

Kotz SA, Ravignani A, Fitch WT (2018) The Evolution of Rhythm Processing. Trends Cogn Sci 22:896-910. https://doi.org/10.1016/j.tics.2018.08.002

Lecanuet JP, Granier-Deferre C, Jacquet AY, Busnel MC (1992) Decelerative cardiac responsiveness to acoustical stimulation in the near term fetus. Q J Exp Psychol B 44:279-303. https://doi.org/10.1080/02724999208250616

Morrongiello BA, Clifton RK (1984) Effects of sound frequency on behavioral and cardiac 
orienting in newborn and five-month-old infants. J Exp Child Psychol 38:429-446. https://doi.org/10.1016/0022-0965(84)90086-9

Nagai Y (2019) Predictive learning: its key role in early cognitive development. Philos Trans R

Soc Lond B Biol Sci 374:20180030. https://doi.org/10.1098/rstb.2018.0030

Patel AD (2014) The evolutionary biology of musical rhythm: was Darwin wrong? PLoS Biol 12:e1001821. https://doi.org/10.1371/journal.pbio.1001821

Peper CLE, Oorthuizen JK, Roerdink M (2012) Attentional demands of cued walking in healthy young and elderly adults. Gait Posture 36:378-382. https://doi.org/10.1016/j.gaitpost.2012.03.032

Perani D, Saccuman MC, Scifo P, et al (2010) Functional specializations for music processing in the human newborn brain. Proc Natl Acad Sci U S A 107:4758. https://doi.org/10.1073/pnas.0909074107

Phillips-Silver J, Trainor LJ (2005) Feeling the beat: movement influences infant rhythm perception. Science 308:1430. https://doi.org/10.1126/science.1110922

Porges SW, Arnold WR, Forbes EJ (1973) Heart rate variability: An index of attentional responsivity in human newborns. Dev Psychol 8:85-92. https://doi.org/10.1037/h0033793

Prechtl HF (1997) State of the art of a new functional assessment of the young nervous system. An early predictor of cerebral palsy. Early Hum Dev 50:1-11. https://doi.org/10.1016/s0378-3782(97)00088-1

Quené H, van den Bergh H (2004) On multi-level modeling of data from repeated measures designs: a tutorial. Speech Commun 43:103-121. https://doi.org/10.1016/j.specom.2004.02.004

R Development Core Team. (2020) R: a language and environment for statistical computing: R Foundation for Statistical Computing.

Repp BH (2005) Sensorimotor synchronization: a review of the tapping literature. Psychon Bull Rev 12:969-992. https://doi.org/10.3758/bf03206433

Richards JE, Casey BJ (1991) Heart Rate Variability During Attention Phases in Young Infants.

Psychophysiology 28:43-53. https://doi.org/10.1111/j.1469-8986.1991.tb03385.x

Rochat P, Striano T (1999) Emerging self-exploration by 2-month-old infants. Dev Sci 2:206-218. https://doi.org/10.1111/1467-7687.00069

Rovee-Collier C (1999) The Development of Infant Memory. Curr Dir Psychol Sci 8:80-85. https://doi.org/10.1111/1467-8721.00019

Savage PE, Brown S, Sakai E, Currie TE (2015) Statistical universals reveal the structures and functions of human music. Proc Natl Acad Sci U S A 112:8987-8992. 
https://doi.org/10.1073/pnas.1414495112

Scherer KR, Zentner MR (2001) Emotional effects of music: Production rules. Music and emotion: Theory and research 487:361-392

Shinya Y, Kawai M, Niwa F, Myowa-Yamakoshi M (2016) Associations between respiratory arrhythmia and fundamental frequency of spontaneous crying in preterm and term infants at term-equivalent age. Dev Psychobiol 58:724-733. https://doi.org/10.1002/dev.21412

Sterling P (2012) Allostasis: a model of predictive regulation. Physiol Behav 106:5-15. https://doi.org/10.1016/j.physbeh.2011.06.004

Sullivan MW (2016) Vagal tone during infant contingency learning and its disruption. Dev Psychobiol 58:366-381. https://doi.org/10.1002/dev.21376

Tarvainen MP, Niskanen J-P, Lipponen JA, et al (2014) Kubios HRV--heart rate variability analysis software. Comput Methods Programs Biomed 113:210-220. https://doi.org/10.1016/j.cmpb.2013.07.024

Task Force of the European Society of Cardiology the North American Society of Pacing (1996) Heart Rate Variability. Circulation 93:1043-1065. https://doi.org/10.1161/01.CIR.93.5.1043

Thelen E (1979) Rhythmical stereotypies in normal human infants. Anim Behav 27:699-715. https://doi.org/10.1016/0003-3472(79)90006-x

Tramo MJ, Lense M, Van Ness C, et al (2011) Effects of Music on Physiological and Behavioral Indices of Acute Pain and Stress in Premature Infants: Clinical Trial and Literature Review. Music Med 3:72

Trappe H-J (2010) The effects of music on the cardiovascular system and cardiovascular health.

Heart 96:1868-1871. https://doi.org/10.1136/hrt.2010.209858

Verschure PFMJ, Pennartz CMA, Pezzulo G (2014) The why, what, where, when and how of goal-directed choice: neuronal and computational principles. Philos Trans R Soc Lond B Biol Sci 369.: https://doi.org/10.1098/rstb.2013.0483

von Hofsten C (2004) An action perspective on motor development. Trends Cogn Sci 8:266-272. https://doi.org/10.1016/j.tics.2004.04.002

Watanabe H, Homae F, Taga G (2011) Developmental emergence of self-referential and inhibition mechanisms of body movements underling felicitous behaviors. J Exp Psychol Hum Percept Perform 37:1157-1173. https://doi.org/10.1037/a0021936

Winkler I, Háden GP, Ladinig O, et al (2009) Newborn infants detect the beat in music. Proc Natl Acad Sci U S A 106:2468-2471. https://doi.org/10.1073/pnas.0809035106

Zatorre RJ, Chen JL, Penhune VB (2007) When the brain plays music: auditory-motor 
interactions in music perception and production. Nat Rev Neurosci 8:547. https://doi.org/10.1038/nrn2152

Zentner M, Eerola T (2010) Rhythmic engagement with music in infancy. Proc Natl Acad Sci U S A 107:5768-5773. https://doi.org/10.1073/pnas.1000121107 